\title{
Distance-dependent switching of anti-predator behavior of frogs from immobility to fleeing
}

\author{
Nozomi Nishiumi • Akira Mori
}

Received: 14 October 2014/ Accepted: 11 December 2014/Published online: 24 December 2014

(C) Japan Ethological Society and Springer Japan 2014

\begin{abstract}
To avoid predation, many animals are required to appropriately switch between immobility for crypsis and fleeing for escape. We conducted two staged-encounter experiments using a frog and a snake to examine factors that affect the occurrence of immobility and fleeing, and to evaluate the efficiency of them. The first experiment demonstrated that frogs initially exhibit immobility, when snakes are moving at a long distance, and then switch from immobility to fleeing at a shorter distance even when snakes have not detected them. The second experiment demonstrated that snakes at $400-800 \mathrm{~mm}$ distance detect only fleeing frogs, whereas snakes at $100 \mathrm{~mm}$ or closer detect both immobile and fleeing frogs. Thus, the ability of snakes to detect motionless frogs depends on the distance, and the distance-dependent switching can be considered an adaptive strategy of the frog. However, a previous model predicts that cryptic prey should flee immediately on seeing a predator or not flee until being detected by the predator. To explain this discordance, we propose two factors: engagement of intensive searching mode by predator at short distance and effects of sudden fleeing at close distance. We suggest incorporating them in future theory for better understanding of anti-predator strategy.
\end{abstract}

Keywords Anti-predator behavior - Anura - Escape decision - Immobility - Optimal flight initiation distance . Predator-prey interaction

N. Nishiumi $(\bowtie) \cdot$ A. Mori

Department of Zoology, Graduate School of Science,

Kyoto University, Sakyo, Kyoto 606-8502, Japan

e-mail: nozo@ethol.zool.kyoto-u.ac.jp

\section{Introduction}

Predation avoidance is an essential process for prey to survive and, thus, prey has evolved to overcome predator (Dawkins and Krebs 1979). In anti-predator mechanisms, prey responds by reducing the probability of successful predation when the prey is located within the mutual perceptual field (Brodie et al. 1991). In this situation, prey animals often engage in a secondary defense phase, which requires appropriate decision making for using anti-predator tactics (Edmunds 1974). The study of decision making for using anti-predator tactics is important for understanding anti-predator mechanisms and, thus, the study has been developed in both theoretical and empirical research fields (Ydenberg and Dill 1986; Ducey and Brodie 1983).

In escape theories, the prey may not always perform anti-predator behavior immediately on seeing the predator, even if the probability of escaping from the predator is reduced by this delay (Ydenberg and Dill 1986). The mechanism underlying the delay is that the prey must often make a trade-off between the cost of being eaten and other potential benefits, such as food acquisition (Ydenberg and Dill 1986). The prey initiates anti-predator responses when the predator comes close to a distance where the cost of being eaten exceeds other potential benefits. Following the economic escape model by Ydenberg and Dill (1986), optimal escape models have been developed which predict the escape decision based on economic considerations about the effects of predation risk, current fitness, and cost of escaping (Cooper and Frederick 2007). In both economic and optimal escape models, fleeing is considered to be a representative anti-predator behavior (Ydenberg and Dill 1986; Cooper and Frederick 2007). Nonetheless, fleeing is not necessarily the only option of anti-predator 
behavior against an approaching predator: some prey animals have a variety of anti-predator behaviors (Burger 1974; Caro 2005; Wasson and Lyon 2005; Ford and Reeves 2008; Toledo et al. 2011). Thus, when these prey animals engage in anti-predator tactics, another trade-off would arise: the prey must choose the most appropriate response among multiple anti-predator behaviors to maximize survival.

In the early stage of the predatory sequence, it is considered that many prey animals exhibit anti-predator behavior that is effective against a searching or approaching predator. A representative anti-predator behavior against a searching predator is immobility, in which the prey becomes motionless to enhance crypsis against visually hunting predators (Edmunds 1974; Endler 1991; Toledo et al. 2011), and one against an approaching predator is fleeing (Endler 1991). It is assumed that if prey has a sufficient head start in fleeing, prey will be able to evade predation, either by outrunning the predator, or by safely reaching a refuge (Broom and Ruxton 2005). However, fleeing from the predator will, in most cases, alert it to the presence of the prey individual. The predator may respond to this detection with attack, which may be successful. Thus, there may be a countervailing pressure for the prey to use immobility. This behavior may allow the prey to survive because the predator may pass by without recognizing the presence of the prey. Therefore, to survive, prey animals must achieve an appropriate balance between immobility and fleeing.

Broom and Ruxton (2005) introduced an optimal switching model of immobility and fleeing. They demonstrated that the optimal strategy for cryptic prey is either fleeing immediately on seeing the predator, or not initiating fleeing until the predator has detected the prey. In addition to this prediction, the model of Broom and Ruxton (2005) provides an important viewpoint: to understand the defensive strategy of prey, it is essential to compare multiple anti-predator tactics and clarify the role of each tactic in the strategy.

In addition to the above theoretical studies, empirical studies of anti-predator strategies also have been conducted. Predictions of escape theories have been confirmed and developed by experimental studies using real prey animals, mainly lizards and crickets (Cooper 1997, 2003; Cooper et al. 2003; Lagos et al. 2014). The repertoires and effectiveness of anti-predator tactics against real predators have also been reported (Wasson and Lyon 2005; Ford and Reeves 2008; Miyatake et al. 2009; Toledo et al. 2011). In spite of the piles of studies reporting anti-predator tactics, studies that compare multiple tactics for clarifying the optimal decision of tactic choice are scarcely explored (but see Ducey and Brodie 1983), especially those from the view point of the optimal tactic-choice model, such as the Broom and Ruxton model (2005).

Frogs are known to be preyed upon by a variety of predators (Duellman and Trueb 1994). They are able to detect a predator by its movement and exhibit defensive behaviors, such as fleeing, immobility, puffing up their body, counterattack and secreting chemicals (Marchisin and Anderson 1978; Toledo et al. 2011). Among them, the most commonly observed defensive behavior is fleeing, followed by immobility (Toledo et al. 2011). Thus, frogs are suitable model animals for examining the switch between immobility and fleeing. In the present study, we focused on the anti-predator strategy of frogs against snakes, which are the most typical predators of frogs (Toledo et al. 2006). We first examined whether frogs switch these tactics optimally, as predicted by the theory of Broom and Ruxton (2005). Then, we experimentally examined how the switching affects survival of the frogs. Finally, based on the results of these experiments, we propose several important factors that should be included in future theoretical models of optimal anti-predator strategy.

\section{General methods}

\section{Study organisms}

The subjects were a ranid frog, Pelophylax nigromaculatus, and a colubrid snake, Elaphe quadrivirgata. Pelophylax nigromaculatus is a pond frog densely distributed over a large part of East Asia, including Japan, Korea, China, and the Amur Basin of Russia (Maeda and Matsui 1999; Shinohara 2007; Zhang et al. 2008). Elaphe quadrivirgata is widely distributed in Japan and is a dietary generalist, mainly feeding on frogs, including $P$. nigromaculatus (Mori and Moriguchi 1988; Kadowaki 1996; Goris and Maeda 2004). Because E. quadrivirgata is diurnal, it is presumed that the snake detects prey mainly by visual cues (Ota 1986).

A total of 124 frogs were used for the experiments. All frogs were collected from Kyoto Prefecture, Japan. They were housed individually in clear plastic terraria $(130 \times 210 \times 160 \mathrm{~mm})$. The floor of each terrarium was slightly inclined, and the terraria contained water that covered half of the floor. The terraria were kept under the natural ambient photoperiod and at air temperature during May-September in Kyoto. During October, the terraria were kept in a laboratory where air temperature was maintained between 25 and $30{ }^{\circ} \mathrm{C}$. Illumination was provided by sunlight. All frogs were used for experiments within 2 weeks after they were captured. Twenty-three 
snakes were used for the experiment (19 and 4 snakes from Kyoto and Tokushima Prefectures, respectively). They were collected from areas sympatric with $P$. nigromaculatus. Snakes were housed individually in clear plastic terraria $(405 \times 265 \times 200 \mathrm{~mm})$ containing glass vessels with water, paper floor and a few pieces of broken plant pots as shelter. The terraria were kept in a laboratory where air temperature was maintained between 25 and $30{ }^{\circ} \mathrm{C}$. Illumination was provided by sunlight. All snakes were fed two or three frogs per week. After the experiment, all frogs were eventually fed to these snakes, except for 15 frogs that were eaten in experiment 2 , and snakes were basically released at the site of capture.

\section{Experimental apparatus}

The test arena, measuring $1,175 \times 452 \times 425 \mathrm{~mm}$, was made of clear glass panels, and set in the center of an experimental room, measuring $4 \times 2 \times 2 \mathrm{~m}$. The arena was divided into two compartments (indoor and outdoor spaces) by a white plastic board (Fig. 1). One edge of this board was attached to a wall of the arena via hinges so that the board could be lifted up with a string. All trials were filmed with a video camera (Canon IVIS HV30) by means of a mirror.

\section{Ethical note}

We conducted all experiments at a laboratory in Kyoto University in compliance with the guidelines of the Animal Care and Use Committee of Kyoto University. Regarding the reduction of sample size, we used the minimum number of animals necessary to achieve the research objectives in all experiments. Concerning the replacement of animals, it

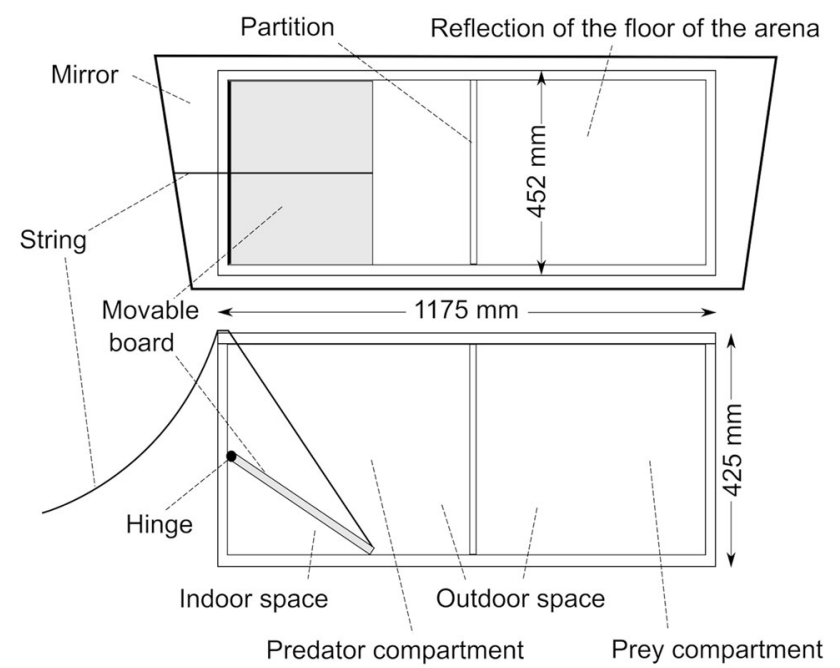

Fig. 1 Top (upper) and side (lower) views of the test arena. See text for detailed descriptions was not suitable to use nonanimal alternatives to examine the efficacy of immobility and fleeing of frogs against predator attack. Thus, we needed to use live frogs and live snakes. Considering the refinement of experimental procedure, we used a partition board to protect frogs from predation by snakes in experiment 1 . However, we did not use the board in experiment 2 , in which the predationavoidance effect of immobility and fleeing, especially at close distance, were examined, because such protections disturb and limit their motion. Consequently, some frogs were eaten by snakes in experiment 2 , but the process was the same as a predation event happening in nature, which did not cause any unnatural pain to the frogs.

\section{Experiment 1: examination of the effects of snake movements and distance between frogs and snakes}

In this experiment, we examined factors that affect the occurrence of immobility and fleeing in encounters between predator and prey. Experiment 1 comprised of two parts: experiments $1 \mathrm{~A}$ and $1 \mathrm{~B}$. Experiment $1 \mathrm{~A}$ was conducted to examine the effects of snake movement on the behavior of frogs. Experiment 1B was conducted to examine the effects of distance between a frog and a snake on the behavior of the frog.

\section{Methods}

In experiment $1 \mathrm{~A}$, the outdoor space of an arena was divided into two compartments: prey and predator compartments, by a clear plastic partition that contained many small holes (Fig. 1). Experiment 1A consisted of three sessions: first we observed the behavior of a frog without a snake (control session-1; CS1), then we observed it with the presence of a snake (experimental session; ES), and finally we conducted a control session again (control session-2; CS2). The same frog was used repeatedly throughout CS1, ES and CS2. The duration of each session was $1 \mathrm{~h}$, and the interval between two successive sessions was 1 day. In ES, we introduced a snake and a frog into the indoor space and the prey compartment of the outdoor space, respectively. Ten minutes after introducing the frog, we lifted the door and recorded the behavior of the snake and frog with the aid of a video camera for $1 \mathrm{~h}$. The distance between a frog and a snake at the start of ES was at least $400 \mathrm{~mm}$. During this session, both frog and snake were allowed to utilize visual and chemical cues through the partition board. In CS1 and CS2, we introduced only a frog into the prey compartment of the outdoor space and recorded its behavior in the same way as in ES. We conducted 18 trials, and each trial contains these three types of 
session. Eighteen frogs and two snakes were used in the experiment. Mean body mass of the frogs was $5.7 \mathrm{~g}$ (range 2.0-19.2 g). Body mass of the snakes was 361 and $478 \mathrm{~g}$, and their snout-vent length was 1,059 and 1,230 $\mathrm{mm}$.

We used C-trax software for analyzing the speed of the locomotive movements of frogs in the video data. C-trax is an open-source program for estimating the positions and orientations of many individual walking flies over long periods of time. It was released by the California Institute of Technology and is designed to allow high-throughput, quantitative analysis of behavior in freely moving flies (http://ctrax.sourceforge.net/). Because the minimum speed of locomotive movements that we were able to recognize was $40 \mathrm{~mm} / \mathrm{s}$, we discarded movements whose speed was less than $40 \mathrm{~mm} / \mathrm{s}$ as noise, and then we counted the number of frog movements. We divided the ES into two periods: when the snake remained motionless (ES-ml) and when it was moving (ES-mv), and then we analyzed the data using the multiple comparison procedure following the Friedman test (Siegel and Castellan 1988), applying treatment (CS-1, CS-2, ES-ml and ES-mv) as the independent variable and the rate of frog movements as the dependent variable.

In experiment $1 \mathrm{~B}$, we introduced a frog and a snake into the indoor space and the outdoor space, respectively, separated from each other by at least $800 \mathrm{~mm}$. The arena did not have a partition, so that the snake was allowed to approach the frog. Ten minutes after introducing them, we lifted the door and recorded their behavior with the aid of a video camera for $1 \mathrm{~h}$. We recorded the timing of the flight initiation of frogs in relation to behavior of snakes and distance between a frog and a snake. We defined the following three responses of E. quadrivirgata to frogs. Phase 1: orienting - a sudden displacement of the head, head and neck, or the anterior part of the body in the direction of the prey. The position of the whole body does not change. Phase 2: straight approaching - slow or rapid locomotion straight toward the prey. Phase 3: striking opening the jaws and projecting the head, head and neck, or the anterior part of the body rapidly toward the prey. We determined whether snakes detected frogs based on the behavior of the snakes. However, it is difficult to judge the occurrence of detection based on orienting behavior because snakes orient not only to frogs but also to many other kinds of objects. Thus, we considered orienting as only an indicator that snakes detected some object(s), and we used straight approaching and striking as an indicator that snakes detected the frogs. Fifty frogs and 13 snakes were used. Mean body mass of the frogs was $4.8 \mathrm{~g}$ (range $0.7-16.6 \mathrm{~g}$ ). Mean body mass and snoutvent length of the snakes were $243 \mathrm{~g}$ (range 25-500 g) and $923 \mathrm{~mm}$ (range 427-1270 $\mathrm{mm}$ ), respectively. No frogs were used more than once.

\section{Results}

In the ES of experiment $1 \mathrm{~A}$, the snake initially remained motionless. Once the snake started moving, it kept moving almost continuously until the end of the session. Mean \pm SD of duration of ES-ml and ES-mv was $18 \pm 14$ and $43 \pm 14 \mathrm{~min}$, respectively $(n=18)$. During the ES, the mean number of movements of the frogs was 49 (range $0-421$ ), and most of the movements were observed while the snake remained motionless (mean 48). The treatments significantly affected the rate of frog movements (Friedman test: $\left.\chi^{2}=21.3, d f=3, P<0.001\right)$. There were no significant differences in the rate of frog movements (the number of movements/hour) among CS-1, CS-2, and ESml (multiple comparison: each $\left|R_{u}-R_{v}\right|<20.43$, each $P>0.05$; Fig. 2). The rate of frog movements when the snake was moving (ES-mv) was significantly lower than that during CS-1, CS-2 and ES-ml (multiple comparison: each $\left|R_{u}-R_{v}\right| \geq 20.43$, each $P<0.05$; Fig. 2).

In experiment $1 \mathrm{~B}$, after the snake started moving, it did not approach the frog directly, but rather crawled around the arena without orienting to the frog. Consequently, the snake shortened the distance between the frog and itself, and in all cases the frogs fled before the snake reached them, and the snake showed neither orienting, straight approaching nor striking before the flight initiation of the

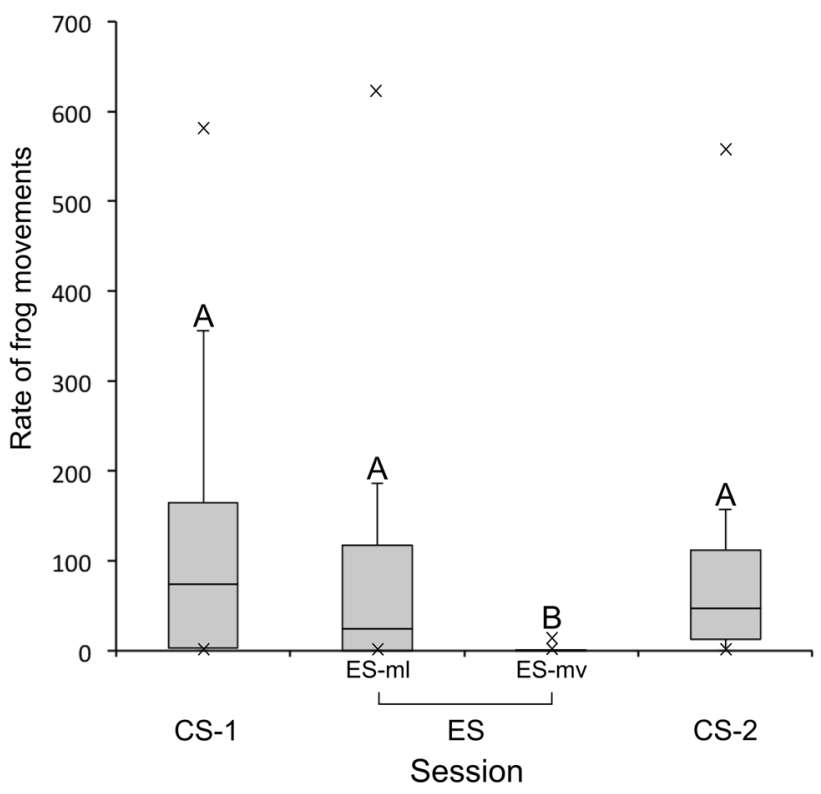

Fig. 2 Box plot of the rate of frog movements (the number of movements per hour). ES is an experimental session in which frog movements are monitored in the presence of a snake. CS is a control session (no snake) conducted before (CS1) or after (CS2) the experimental session. ES-ml and ES-mv indicate the period when the snake remained motionless and when the snake was moving, respectively. Different letters above the boxes indicate significant differences (multiple comparison: $\left|R_{u}-R_{v}\right| \geq 20.43, P<0.05$ ) 
frogs. Mean \pm SD of the distance between the snake and the frog when the latter exhibited the first fleeing was $80 \pm 67 \mathrm{~mm}(n=50$; Fig. 3$)$.

\section{Discussion}

The lack of a significant difference between CS-1 and CS-2 in experiment $1 \mathrm{~A}$ indicates the absence of acclimation effects. The rate of frog movements during ESmv being significantly lower than the others indicates that frogs detect a snake through its movement. The results of experiment $1 \mathrm{~B}$ showed that frogs first become immobilized and then switch to fleeing according to their distance from a snake, which is before being detected by the snake. Overall, the results of experiment 1 suggest that frogs recognize the predation threat of a snake by its movement always before they are detected by the snake, which is the basic assumption of the model of Broom and Ruxton (2005). Nonetheless, contrary to their predation, frogs switch from immobility to fleeing at an intermediate time between detecting a snake and being detected by it.

\section{Experiment 2: examination of the effectiveness of immobility in relation to distance}

In experiment 1 , frogs switched from immobility to fleeing according to their distance from snakes. This suggests the increase of predation risk with decreasing distance to a predator. We assumed that the probability of detection of motionless frogs by snakes might increase as the distance between them becomes short. To test this assumption, we examined the effectiveness of immobility against snakes at two different distances.

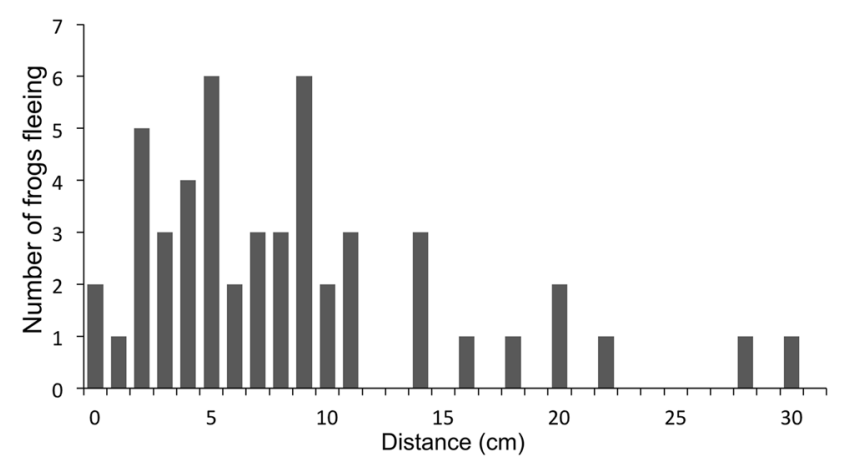

Fig. 3 Frequency distribution of the distance between a frog and a snake when the frog started fleeing in response to the approaching snake

\section{Methods}

We examined the responses of snakes against a motionless and a moving frog at two different distances: short distance $(0-100 \mathrm{~mm})$ and long distance (400-800 mm). The responses of snakes were defined as experiment $1 \mathrm{~B}$.

We introduced a frog and a snake into the indoor space and the outdoor space, respectively, separated from each other by at least $800 \mathrm{~mm}$. The arena did not have a partition, so that the snake was allowed to approach the frog. Ten minutes after introducing them, we lifted the door and recorded their behavior with the aid of a video camera for $1 \mathrm{~h}$. After the snake started moving, the snake usually explored the arena, shortening its distance from the frog. In the test of snakes against a motionless frog, we observed the snake's behavior toward a motionless frog when the snake was crawling within the range of $400-800 \mathrm{~mm}$ (session of motionless frogs at long distance; session-ml-L) and within the range of $0-100 \mathrm{~mm}$ (session of motionless frogs at short distance; session-ml-S). In the test of snakes against a moving frog, we observed the snake's behavior toward a moving frog when the snake was crawling within the range of 400-800 $\mathrm{mm}$ (session of moving frogs at long distance; session-mv-L) and within the range of 0-100 $\mathrm{mm}$ (session of moving frogs at short distance; session-mv-S). We terminated the sessions when the snake moved out of the distance range without showing straight approaching or when the snake struck the frog.

According to the results of experiment 1B, within the long-distance range, frogs usually exhibited immobility and did not voluntarily move, and within the short-distance range, frogs usually exhibited fleeing as a response to an approaching snake. Thus, we used frogs without manipulation in session-ml-L and session-mv-S. However, we needed to manipulate frogs to initiate moving in session$\mathrm{mv}-\mathrm{L}$, and to inhibit moving in session-ml-S. To induce the frogs to move within the long-distance range, a string was tied around the bellies of the frogs. By pulling the string from outside of the arena, we induced the frogs to perform locomotive movement similar to voluntary jumping. After the snake reached the long-distance range, we pulled the string basically once every $5 \mathrm{~s}$ until the snake struck the frog. On the other hand, to prevent frogs from fleeing within the short-distance range, we lowered their body temperature because frogs are not able to move at low body temperatures. We soaked the frogs in ice water for $15 \mathrm{~min}$ and then put them on an ice pack $(100 \mathrm{~mm} \times$ $100 \mathrm{~mm} \times 10 \mathrm{~mm}$ ) on the floor of the arena and kept them there during the session.

In session-ml-L, 20 snakes and 20 frogs were used. Mean body mass of these frogs was $5.0 \mathrm{~g}$ (range 4.3-12.7 g). Mean body mass and snout-vent length of these snakes were $219 \mathrm{~g}$ (range 25-500 g) and $915 \mathrm{~mm}$ 
(range 427-1,270 $\mathrm{mm}$ ), respectively. In session-ml-S, nine snakes and nine frogs were used. Mean body mass of these frogs was $2.1 \mathrm{~g}$ (range 1.1-5.0 g). Mean body mass and snout-vent length of these snakes were $179 \mathrm{~g}$ (range $42-355 \mathrm{~g}$ ) and $874 \mathrm{~mm}$ (range 530-1,120 $\mathrm{mm}$ ), respectively. In session-mv-L, seven snakes and seven frogs were used. Mean body mass of these frogs was $8.3 \mathrm{~g}$ (range $2.4-12.1 \mathrm{~g}$ ). Mean body mass and snout-vent length of these snakes were $166 \mathrm{~g}$ (range 112-320 g) and $869 \mathrm{~mm}$ (range 720-1,120 mm), respectively. In session-mv-S, 20 snakes and 20 frogs, which were the same individuals as those in session-ml-L, were used. No frog was used more than once, but seven snakes were used in both session-ml-L and session-mv-L, and in both session-mv-S and session$\mathrm{ml}-\mathrm{S}$.

We analyzed the data of experiment 2 using GLMM with binomial family, applying the occurrence of detection of the frog as the dependent variable, the distance between the frog and the snake at the start of each session (long or short) as a fixed factor, and the snake's ID as a random factor. The statistical package JMP (version, 8.0.2) was used for the GLMM analyses.

\section{Results}

In the test of snakes against a motionless frog, no snakes showed orienting, straight approaching or striking against the frog at the long distance. On the other hand, at the short distance all snakes approached the motionless frog slowly but not on a straight path, while frequently flicking their tongues, and eventually contacted the frog with their snouts. Eight of the nine snakes then grasped the frog with their jaws (striking). The other snake did not exhibit striking and instead resumed crawling. The distance significantly affected the occurrence of predatory behavior of snakes $\quad$ (GLMM: $\quad$ coefficient $=0.44, \quad t=12.21$, $P<0.0001)$.

In the test of snakes against a moving frog, all snakes immediately exhibited orienting to the frog at the long distance, and then performed straight approaching and finally struck the frog (Table 1). On the other hand, against the moving frog at the short distance, although all snakes immediately exhibited orienting to it, only $70 \%$ of the

Table 1 The number of snakes that detected frogs in experiment 2

\begin{tabular}{lll}
\hline Experiment & \multicolumn{2}{l}{ Behavior of frogs } \\
\cline { 2 - 3 } & Motionless & Moving \\
\hline Long distance $(400-800 \mathrm{~mm})$ & $0(20)$ & $7(7)$ \\
Short distance $(0-100 \mathrm{~mm})$ & $8(9)$ & $14(20)$
\end{tabular}

Numerals in parentheses are the total number of trials snakes performed straight approaching and struck it. The remaining $30 \%$ of the snakes performed orienting but exhibited neither straight approaching nor striking, and then resumed crawling. The distance did not significantly affect the occurrence of predatory behavior of snakes (GLMM: coefficient $=-0.15, t=-1.67, P=0.108)$.

\section{Discussion}

The present results demonstrated that immobility of the frog was effective for avoiding detection by snakes only at a long distance. When the snakes were positioned at a close distance, the frog was not able to avoid detection regardless of their anti-predator behaviors. This suggests that the snakes engage in another searching mode at a short distance, which enables them to detect frogs eventually without a movement cue, because behavior exhibited by the snakes approaching the motionless frog at the short distance seemed quite different from that at the long distance (see General discussion). On the other hand, the snakes struck the moving frog in all cases at the long distance, whereas $30 \%$ of snakes showed neither straight approach nor strike against moving frogs at short distance. This implies that sudden movement of frogs at close distance may suppress predatory response of snakes to some extent (See also below).

\section{General discussion}

In the model of Broom and Ruxton (2005), it was demonstrated that the optimal strategy for prey is either fleeing immediately on seeing the predator or not initiating fleeing until the predator detects the prey. The former strategy has the advantage that the prey can initiate fleeing at the maximum distance, and the timing of the initiation of fleeing is before the prey is detected, when the predator may not be able to respond to it immediately. On the other hand, the latter strategy has an advantage that the predator may pass without detecting the prey. In addition, Broom and Ruxton (2005) demonstrated that it is never optimal for prey to use immobility first, and then initiate fleeing after waiting for the predator to reach a certain distance, but before being detected. In this "inappropriate" strategy, the prey abandons the advantage of crypsis and initiates fleeing at a shorter distance than that of immediate fleeing. However, in contradiction to this model, the frogs in experiment 1B responded with the "inappropriate" strategy: they remained motionless when they first noticed the moving snake, and then they initiated fleeing at a certain distance before the snake obviously detected them. There are at least two possible explanations for this unexpected result. 
First, when frogs initiated fleeing, although the snakes had not detected the frogs, future detection may have been no longer avoidable if the frogs remained motionless. The model of Broom and Ruxton (2005) is based on the assumption that there is still possibility that the predator will pass by the prey without detecting it if the predator has not detected it. However, if the predator engages in a searching mode that enables it to eventually detect the prey within a certain range, the circumstance of unavoidable future detection would occur. Immobility is a cryptic tactic that is effective against predators using a visual sense, and it may not work in avoiding detection by other senses, especially at a short distance. In our experiments, snakes were able to detect immobile frogs within $100 \mathrm{~mm}$ distance even in the absence of the movement cue. It is well known that snakes have a keen chemical sense that relies on the vomeronasal organ (Jacobson's organ) and that they are able to detect prey with chemical cues alone (Halpern 1987; Wattiez et al. 1994). Thus, it is likely that E. quadrivirgata used some chemical cue(s) for detecting nearby frogs. Although chemical cues are not effective in locating the exact position of remote frogs, intensive local search using chemical cues would enable snakes to eventually detect the prey in the vicinity. Therefore, even when snakes have not detected frogs, the frogs should initiate fleeing at a distance that they are expected to be detected at sooner or later. The occurrence of the intensive searching mode at short distance, which leads to definite detection of prey, may be one possible explanation of the discordance between our results and the prediction of Broom and Ruxton (2005).

Second, the relationship between the distance and the probability of successful escape by fleeing may not be simple. The theory of Broom and Ruxton (2005) is based on a presumption that the probability of successful escape by fleeing decreases as the distance between the prey and the predator decreases because they simply considered that the function of fleeing is to increase the distance between prey and predator. However, contrary to this presumption, close distance may add other functions to fleeing. For example, when prey initiates fleeing at a shorter distance, the angle between the longitudinal axis of the head of the predator and the line from the predator to the prey changes rapidly. Consequently, the predator may not be able to keep track of the moving prey. Moreover, the predator may not be able to immediately recognize the moving object at close proximately as prey or the predator may be frightened by the sudden movement of the prey (Gamberale-Stille et al. 2009). Indeed, $30 \%$ of the frogs in experiment 2 that initiated fleeing at short distance did not induce immediate attack of the snakes. Therefore, initiating fleeing at short distance may provide an additional defensive function, resulting in a lower predation risk than fleeing at an intermediate distance. This may be the reason why the frogs did not flee immediately when they recognized the snakes, but subsequently initiated fleeing before being detected.

In summary, we propose two new viewpoints for understanding the interplay between predator and prey. First, at a short distance, some predators switch to intensive searching mode with the aid of additional sensory cues, which leads them to eventually detect the prey. Against such predators, the adaptive response of prey at a short distance is to start fleeing even before the predator detects it. This presumption has not been considered in the model by Broom and Ruxton (2005), in which prey still has a chance of letting the predator pass without being detected. Second, short distance can create an additional defensive function of fleeing. We call this effect the "close-quarters effect". The close-quarters effect would apply not only to the frog-prey and snake-predator system, but also to many other animals. For example, as a butterfly opens its wings with eyespots against predator in order to flee, the deimatic impact would be enhanced at short distance (Gamberale-Stille et al. 2009; Vallin et al. 2005). In studies of anti-predator strategy, the probability of escaping predation by fleeing has been simply assumed as a monotonically increasing function of distance between a prey and its predator. However, it is highly likely that another distance-dependent effect, such as the closequarters effect, would partly change the shape of the function from monotonically increasing to convex upward, resulting in higher escape probability of fleeing at proximate distance. Although the present study was conducted under simplified environmental conditions, and examination in a more natural setting would be required, we anticipate that incorporating the above two points will contribute to better understanding of the anti-predator strategy of animals in the real world.

Acknowledgments We thank D. Muramatsu and T. Jono for their advice on statistical analysis and E. Nakajima for kindly reading and improving the manuscript. We also thank N. Fuse and K. Tsujimoto for allowing us to use their computer software for analyzing C-trax data. This work was partially supported by the Global COE Program (A06) and Grants for Biodiversity \& Evolution Project of Excellent Graduate Schools to Kyoto University from MEXT, Japan, and the Sasakawa Research Grant (26-528) to Nozomi Nishiumi from the Japan Science Society.

\section{References}

Brodie ED Jr, Formanowicz DR Jr, Brodie IIIED (1991) Predator avoidance and antipredator mechanisms: distinct pathways to survival. Ethol Ecol Evol 3:73-77

Broom M, Ruxton GD (2005) You can run —or you can hide: optimal strategies for cryptic prey against pursuit predators. Behav Ecol 16:534-540 
Burger J (1974) Breeding adaptations of Franklins gull (Larus pipixcan) to a marsh habitat. Anim Behav 22:521-567

Caro T (2005) Antipredator defenses in birds and mammals. University of Chicago Press, Chicago

Cooper WE (1997) Factors affecting risk and cost of escape by the broad-headed skink (Eumeces laticeps): predator speed, directness of approach, and female presence. Herpetologica $53: 464-474$

Cooper WE (2003) Effect of risk on aspects of escape behavior by a lizard, Holbrookia propinqua, in relation to optimal escape theory. Ethology 109:617-626

Cooper WE, Frederick WG (2007) Optimal flight initiation distance. J Theor Biol 244:59-67

Cooper WE, Pérez-Mellado V, Baird TA, Baird TD, Caldwell JP, Vitt LJ (2003) Effects of risk, cost, and their interaction on optimal escape by nonrefuging Bonaire whiptail lizards, Cnemidophorus murinus. Behav Ecol 14:288-293

Dawkins R, Krebs JR (1979) Arms races between and within species. Proc R Soc Lond B 205:489-511

Ducey PK, Brodie ED Jr (1983) Salamanders respond selectively to contacts with snakes: survival advantage of alternative antipredator strategies. Copeia 1983:1036-1041

Duellman WD, Trueb L (1994) Biology of amphibians. McGraw-Hill, New York

Edmunds M (1974) Defence in animals: a survey of anti-predator defences. Longman, London

Endler JA (1991) Interactions between predators and prey. In: Krebs JR, Davies NB (eds) Behavioural ecology: an evolutionary approach. Blackwell Scientific, Oxford, pp 169-196

Ford JKB, Reeves RR (2008) Fight or flight: antipredator strategies of baleen whales. Mamm Rev 38:50-86

Gamberale-Stille G, Bragée C, Tullberg BS (2009) Higher survival of aposematic prey in close encounters with predators: an experimental study of detection distance. Anim Behav 78:111-116

Goris RC, Maeda N (2004) Guide to the amphibians and reptiles of Japan. Krieger Publishing, Florida

Halpern M (1987) The organization and function of the vomeronasal system. Annu Rev Neurosci 10:325-362

Kadowaki S (1996) Ecology of a Japanese snake communityresource use patterns of the three sympatric snakes, Rhabdophis tigrinus, Elaphe quadrivirgata and Agkistrodon b. blomhoffii. Bull Tsukuba Univ For 12:77-148 (in Japanese)
Lagos A, Ebensperger LA, Herberstein ME (2014) A quantitative test of the 'economic' and 'optimal' models of escape behaviour. Anim Behav 97:221-227

Maeda N, Matsui M (1999) Frogs and toads of Japan (revised ed). Bun-ichi Sogo Shuppan, Tokyo

Marchisin A, Anderson JD (1978) Strategies employed by frogs and toads (Amphibia, Anura) to avoid predation by snakes (Reptilia, Serpentes). J Herpetol 12:151-155

Miyatake T, Nakayama S, Nishi Y, Nakajima S (2009) Tonically immobilized selfish prey can survive by sacrificing others. Proc R Soc B 276:2763-2767

Mori A, Moriguchi H (1988) Food habits of the snakes in Japan: a critical review. Snake 20:98-113

Ota H (1986) Snake really an able hunter? Predatory behavior of Japanese striped snake, Elaphe quadrivirgata, in the field. J Ethol 4:69-71

Shinohara N (2007) The effect of the new-style of rice paddies on the distribution in Rana nigromaculata, Kagawa Prefecture, Japan. Kagawa Seibutsu 34:97-105 (in Japanese)

Siegel S, Castellan NJ (1988) Non-parametric statistics for the behavioral sciences, 2nd edn. McGraw-Hill, New York

Toledo LF, Silva RR, Haddad CFB (2006) Anurans as prey: an exploratory analysis and size relationships between predators and their prey. J Zool 271:170-177

Toledo LF, Sazima I, Haddad CFB (2011) Behavioural defences of anurans: an overview. Ethol Ecol Evol 23:1-25

Vallin A, Jakobsson S, Lind J, Wiklund C (2005) Prey survival by predator intimidation: an experimental study of peacock butterfly defence against blue tits. Proc R Soc B 272:1203-1207

Wasson K, Lyon BE (2005) Flight or fight: flexible antipredatory strategies in porcelain crabs. Behav Ecol 16:1037-1041

Wattiez R, Remy C, Falmagne P, Toubeau G (1994) Purification and preliminary characterization of a frog-derived proteinaceous chemoattractant eliciting prey attack by checkered garter snakes (Thamnophis marcianus). J Chem Ecol 20:1143-1160

Ydenberg RC, Dill LM (1986) The economics of fleeing from predators. Adv Stud Behav 16:229-249

Zhang H, Yan J, Zhang G, Zhou K (2008) Phylogeography and demographic history of Chinese black-spotted frog populations (Pelophylax nigromaculata): evidence for independent refugia expansion and secondary contact. Evol Biol 8:21-36 